\title{
La institucionalización de la educación en derechos humanos en América Latina: avances, desafíos y una propuesta de prioridades
}

\author{
Ana María Rodino \\ Doctora en Educación pela Universidad de Harvard. \\ Investigadora de la Universidad Estatal a Distancia en Costa Rica \\ Profesora en la Universidad de Buenos Aires \\ Profesora en la Universidad de La Plata \\ Buenos Aires, Argentina \\ anamariarodino@gmail.com
}

\begin{abstract}
Resumen Tomando en consideración las conclusiones de investigaciones recientes sobre la incorporación de la Educación en Derechos Humanos en los sistemas escolares de América Latina, el artículo resume los avances alcanzados durante el período 2000/2012, así como también las carencias y limitaciones que subsisten. Sobre esa base y a fin de enfrentar los múltiples desafíos por delante, la autora recomienda alcanzar acuerdos programáticos entre expertos, practicantes e instituciones que trabajan en educación en derechos humanos en la región y hace una serie de propuestas estratégicas que considera prioritarias por su urgencia, integralidad e impacto multiplicador. Las propuestas en materia de educación en derechos humanos se centran en las áreas de investigación,pedagogía, currículo, gestión y práctica escolar, y políticas públicas. En relación a cada área se hace una breve estimación de su estado actual, se recomiendan prioridades de acción, y se plantean preguntas a las cuales es importante dar respuesta.
\end{abstract}

Palabrasclave: educación en derechos humanos, América Latina, investigación educativa, pedagogía de la educación en derechos humanos, currículo, gestión escolar, políticas públicas educativas.

\section{Lo hecho: resultados y conclusiones de la investigación y la experiencia}

Comenzaré haciendo una revisión rápida de las conclusiones disponibles sobre el estado actual de la educación en derechos humanos en América Latina. Intento una mirada regional, apoyándome en la feliz circunstancia de trabajar muy de cerca con un organismo de alcance regional. Me baso en:

- Resultados del investigaciones en las que participé para el Instituto Interamericano de Derechos Humanos:

- Los 10 Informes Interamericanos de la educación en derechos humanos, en particular los 5 últimos por ser los más recientes (desde 2007 hasta 2011) ${ }^{1}$ y

1. Todos las publicaciones del Instituto Interamericano de Derechos Humanos citadas aquí y referenciadas al final del artículo pueden consultarse en www.iidh.ed.cr 
- El esfuerzo de consulta directa a educadores que se llamó Consulta Interamericana sobre la educación en derechos humanos (entre 2012 y 2013), la cual representó una especie de "informe final de la serie de los 10 Informes de la educación en derechos humanos" que incluyó síntesis, actualización y comentario de sus aportes (ver Instituto Interamericano de Derechos Humanos, 2013).

- Mi experiencia directa de estudio, enseñanza, trabajo de campo compartido y discusión permanente con colegas de distintos países de nuestra región.

Estas conclusiones son recogidas y analizadas en detalle en Rodino (2014). Aquí me limitaré a un punteo somero de ellas, que traigo a colación porque son la base de apoyo para las propuestas que plantearé después. Son útiles porque ofrecen un panorama amplio de las tendencias continentales apoyado en evidencias documentales. Nos dicen "por dónde andan" los esfuerzos de conjunto; aunque debo aclarar que no aluden a ningún país en especial por lo cual deben ser "situadas" o "contextualizadas" para cada país.

\section{Desarrollos 2000-2012}

Progresos en la normativa y la institucionalidad educativa (medición de 2007; Instituto Interamericano de Derechos Humanos, 2007)

1. Hubo una inclusión gradual de la educación en derechos humanos como meta explícita en la legislación educativa nacional de la región (constituciones, leyes federales de educación, decretos y resoluciones de los ministerios de educación). En la actualidad, esa inclusión es unánime.

2. En forma paralela al crecimiento normativo se crean dependencias específicas (direcciones o coordinaciones) para atender la capacitación, promoción y acciones particulares en derechos humanos en ministerios y otras entidades gubernamentales, en el ámbito educativo y otros (cultura, justicia, seguridad, etc.)

3. En la legislación específicamente educativa, se adoptaron (o se reformaron para profundizarlas) normas que reconocen organizaciones del tipo "gobiernos estudiantiles", entendidos como espacios de participación, representación, deliberación y decisión del estudiantado dentro de los centros escolares. O sea, espacios potenciales para ejercer y aprender derechos humanos.

Progresos -y limitaciones- en los conocimientos específicos de derechos humanos dentro de los planes y programas de estudio (medición de 2008; Instituto Interamericano de Derechos Humanos, 2008)

4. Se incorporan de modo creciente temas relacionados con los derechos humanos y la democracia, sus principios y valores, en el currículo de primaria y secundaria y en más grados del sistema.

5. Se destaca la presencia, amplitud y profundidad de contenidos de estudio sobre la diversidad (étnica, religiosa, lingüística y cultural), su valor para la sociedad y el rechazo de toda forma de discriminación. Sin embargo, todavía es escasa la referencia a la diversidad de orientación sexual.

6. No obstante, las nutridas referencias a los derechos humanos y la democracia parecen más nominales o simbólicas que sustanciales, debido a varios patrones que predominan:

6.1. Los programas no incluyen conceptualizaciones comprehensivas de los derechos humanos, la democracia y el Estado de Derecho que tanto se nombran (por ej. sus definiciones, principios y fundamentos, rasgos característicos, distintas interpretaciones y sus riesgos, etc.). Hay debilidad de contenidos institucionales.

6.2. Los programas son débiles en información y análisis histórico (cómo la humanidad construyó las nociones de democracia y derechos humanos y cómo evolucionaron en el tiempo, sus hitos históricos y los eventos cruciales en las luchas por los derechos humanos en el mundo, el continente y cada país).

6.3. Los programas no mencionan (o lo hacen muy poco) a personas relevantes que lucharon por los derechos humanos y la democracia, en la historia y en la actualidad, en el mundo el continente y cada país (militantes, pensadores, políticos, activistas comunitarios etc.)

6.4. Los programas no abordan (o lo hacen de manera escasa y ocasional) casos de violaciones masivas de derechos humanos en la historia reciente del mundo, el continente y cada país (en lo que aplique).

7. Prevalece ampliamente la noción tradicional de "ciudadanía" asociada a la nacionalidad y a la pertenencia a una comunidad dentro de un territorio cerrado y con una cultura homogénea. Hay muy poca presencia del concepto moderno, más universal y plural de "ciudadanía global", que plantea que somos ciudadanos del globo y debemos reconocer nuestras responsabilidades hacia cada uno de nuestros semejantes y nuestro hábitat común, el planeta Tierra.

8. Se pone mayor énfasis en el estudio de las normas de derechos humanos que el de las instituciones encargadas de protegerlos -nacionales y supranacionales.

Progresos -y limitaciones- en los conocimientos específicos de derechos humanos en los libros de texto (medición de 2009, ver Instituto Interamericano de Derechos Humanos, 2009).

9. En conjunto, en la década de 2000 los libros escolares estudiados muestran un crecimiento claro y sostenido en el 
abordaje de conocimientos sobre derechos humanos, paralelo $y$ similar al de los programas de estudio.

10. En contraste con los programas de estudio, los libros escolares tratan de modo más amplio dos importantes aspectos de contenido: (a) antecedentes históricos y (b) referencia a personas relevantes en la lucha por los derechos humanos. El tratamiento no es profundo sino apenas moderado, pero es mayor que lo que piden los programas y además, va creciendo en la década.

11. En los programas de estudio y en los libros escolares, muchas problemáticas sociales no se analizan relacionadas con los derechos humanos, aunque sí lo están (por ej. las migraciones y desplazamientos, la pobreza, la sostenibilidad del desarrollo, la salud sexual y reproductiva y la violencia comunitaria e intrafamiliar). No se logra construir todavía un "enfoque" o "perspectiva de derechos" para mirar e interpretar la realidad social.

Progresos en la metodología de educación en derechos humanos en los libros de texto (medición de 2010 a partir de los ejercicios y actividades prácticas para estudiantes; ver Instituto Interamericano de Derechos Humanos, 2010).

12. Se produjo un crecimiento doble en los ejercicios para estudiantes durante la década: aumentó el total de ejercicios de los libros y, aparte, aumentó la proporción de ejercicios explícitos sobre derechos humanos.

13. Los ejercicios explícitos sobre derechos humanos incluyen, además de información en la materia,presencia creciente entre 2000-2010 de (a) valores y actitudes de derechos humanos y (b) destrezas o capacidades para actuar a favor de los derechos humanos.

14. Al analizar el diseño global de los ejercicios como actividad pedagógica (es decir, cómo se estructuran y qué técnicas y recursos emplean) se observa un aumento importante de estrategias que movilizan la participación del estudiantado.

La dimensión de las interacciones: convivencia en la escuela $y$ formación docente (para convivencia, medición de 2010, ver Instituto Interamericano de Derechos Humanos, 2011; para formación de educadores, medición de 2005, ver Instituto Interamericano de Derechos Humanos, 2005)

15. Desde la segunda mitad de 2000 y acelerándose a partir de 2008, se comprueba una tendencia a diseñar políticas de convivencia y seguridad escolar con enfoque de derechos (por ej. programas y reglamentos de convivencia; proyectos de prevención de violencias en la escuela y protocolos para atender sus manifestaciones específicas - acoso escolar o "bullying", agresiones, vandalismo, portación de armas de fuego, tráfico de drogas, etc.-). La mayoría de estas politicas asigna un lugar importante a la educación en derechos humanos y la cultura de paz dentro de las aulas y del centro escolar.

16. La formación de docentes sigue siendo el eslabón más débil para la educación en derechos humanos. Los programas de formación inicial (en su mayoría ofrecidos por universidades) aún prestan poca o ninguna consideración en sus planes de estudio a la pedagogía de los derechos humanos; los de actualización en servicio (generalmente ofrecidos por los ministerios de educación) consisten en actividades breves, ocasionales y de reducida cobertura, muy variables de país a país.

\section{Lo por hacer: desafíos numerosos y muy variados}

Mirando al futuro, permítanme remarcar algo: para hacer progresar la educación en derechos humanos en la educación formal hay que actuar sobre el sistema educativo completo, viéndolo como un todo, donde cada componente cumple una función, hace aportes específicos y también tiene limitaciones propias. Todos los componentes son importantes y en todos hay que introducir el enfoque de derechos.

El componente normativo legitima a la educación en derechos humanos como un compromiso del Estado y una política pública y así es condición de posibilidad para que ella entre a la escuela. El currículo explicita los contenidos y orientaciones pedagógicas de educación en derechos humanos que deben regir en la escuela y así es condición de posibilidad para que ella llegue a las aulas. Los libros escolares hacen el desarrollo extenso de los contenidos de educación en derechos humanos tal como los estudiarán y ejercitarán los maestros y alumnos, y por eso es condición de posibilidad para llevar el enfoque de derechos de manera efectiva a la formación de los niños y niñas de todo un país.

También ocupa un lugar clave la formación de los educadores, porque las normas, el currículo y los libros escolares no actúan solos sino a través de agentes personales, quienes en realidad conducen el proceso de enseñanza-aprendizaje. E igualmente determinantes son las prácticas cotidianas del centro escolar: la cultura de gestión y de convivencia que impera en la escuela, pues ella puede apoyar o contradecir lo que se enseña formalmente.

Los resultados que obtenga el sistema educativo son el producto de la labor articulada y combinada de todos sus componentes, sin protagonismos ni exclusiones. Así pues, a todos ellos tenemos que llegar con la educación en derechos humanos. El desafío que tenemos por delante es amplio, retador y nos convoca a seguir avanzando sin pausa.

¿Cómo evaluar lo hecho y encarar lo por hacer? Aquí voy a hacer una generalización a partir de mi experiencia personal. Percibo que cuando se consulta con personas que trabajan directamente en el campo-educadores formales y no formales, estudiosos y gestores de proyectos en educación en derechos humanos-, uno encuentra que: 
a) Lo hecho local o focalizadamente por las organizaciones y personas consultadas se juzga satisfactorio o muy satisfactorio (en relación a quienes fueron sus beneficiarios directos y dentro de su campo específico de acción), pero...

b) Los impactos más amplios no se pueden evaluar con claridad, aunque la visión de lo que podría estar ocurriendo es insatisfactoria (en la ciudad, el Estado o provincia y, por supuesto, el país). Se siente que la incidencia en círculos más amplios es escasa o se desconoce; que tiene debilidades y limitaciones; que hay dudas sobre su sostenibilidad autónoma (propia, sin apoyos) en el tiempo

c) Los desafíos a futuro se sienten gigantescos. El análisis prospectivo tiende a ser desalentador, a veces desesperanzado;las tareas por delante son muchas y algunas se sienten titánicas (por la gran cantidad de destinatarios a los que hay que llegar; por los largos plazos de ejecución que demandan; por la suma de voluntades que exigen, voluntades políticas y técnicas; por las dificultades de garantizar la sostenibilidad de las iniciativas a mediano y largo plazo...)

Es comprensible. Los educadores somos esencialmente gente de acción y la educación en derechos humanos nos convoca a la acción transformadora. Pero la transformación que buscamos -económico-social, política, cultural- es tan profunda que difícilmente podemos satisfacernos con lograr cambios individuales o de pequeños grupos, y muy localizados. Sin embargo, en este compromiso no podemos permitirnos la desesperanza, el escepticismo o la frustración...

Por ello, mi posicionamiento - provisional y abierto a la discusión- es que tenemos que pensar de manera estratégica y, deseablemente, alcanzar acuerdos programáticos para trabajar en una misma dirección para que los esfuerzos -individuales y de nuestras instituciones - se sumen.

Por acuerdo programático entiendo la concertación de acciones entre dos o más sectores $y / 0$ entidades, para la ejecución de un programa determinado, en las cuales se especifican las actividades que coordinadamente deben darse entre las áreas involucradas para el cumplimiento de los planes y programas. ${ }^{2}$

Lo segundo (alcanzar acuerdos programáticos) es mucho más difícil que lo primero (pensar de manera estratégica).Porque pensar se puede hacer solo o en pequeños grupos, pero alcanzar acuerdos y cumplir los exige concertar muchas voluntades. Por eso, creo, es que en la región ha costado tanto elaborar Planes nacionales de educación en derechos humanos verdaderamen- te intersectoriales y, a pesar del tiempo transcurrido desde la convocatoria de la Organización de Naciones Unidas al respecto (1995), hay tan pocos en ejecución. Pero el tema de los Planes nacionales de educación en derechos humanos merece otra discusión separado.

Aquí quiero ensayar hacer algunas (pocas) propuestas estratégicas para el trabajo futuro en educación en derechos humanos. Aclaro que no me referiré al derecho a la educación porque el foco de este trabajo es la educación en derechos humanos. No obstante, sugiero que los educadores en derechos humanos debemos también contemplar esa dimensión sustancial, ya que sin su vigencia efectiva no hay posibilidad de hacer educación en derechos humanos.

\section{Lo prioritario: Propuestas estratégicas}

El eje de este trabajo es deliberadamente desafiante. Voy a proponer cinco prioridades para el trabajo de educación en derechos humanos en América Latina en el momento actual. Las considero estratégicas porque estoy convencida que son:

- urgentes (acciones necesarias para nuestro contexto ya)

- abarcadoras (con influencia en múltiples niveles del sistema educativo)

- multiplicadoras o, con más precisión, formadoras de agentes multiplicadores (en este sentido son, utilizando el concepto de Amartya Sen, constructoras de agencia, por lo cual poseen gran posibilidad de impacto (Sen, 1985).

Acepto el riesgo y la crítica de dejar mucho por fuera, pero lo hago con la convicción de que "priorizar todo es no priorizar nada". Según la información de que dispongo, creo que estas prioridades se aplican a la mayoría, sino a todos, los países latinoamericanos. Cada lector evaluará si esto es aplicable a su respectivo contexto y en qué medida, o debe ser adaptado

Las prioridades que señalo corresponden a distintos ámbitos. Por lo tanto, implican mucho trabajo para muchas personas que se desempeñan en diferentes espacios de la educación. O sea, son muy incluyentes porque suman diversas tareas y diversos actores.

\subsection{Investigación en educación en dere- chos humanos}

Estimación del estado actual:

Se encuentran trabajos teóricos (propositivos) y de presentación de experiencias específicas (descrip- 
tivos), pero pocos estudios nacionales y, menos aún, estudios de campo.

\section{Prioridad:}

(1) Investigación descriptiva de cobertura nacional:

Realizar diagnósticos nacionales que permitan orientar políticas públicas de educación en derechos humanos(por ej. apoyar la elaboración de un PLANEDH nacional o su actualización;ampliar la incorporación de la educación en derechos humanos en los currículos de la educación formal;incluir educación en derechos humanos en la formación de servidores públicos).

(2) Investigación de campo y transdisciplinaria:

Evaluar y compartir resultados, conclusiones y "lecciones aprendidas" sobre las experiencias de educación en derechos humanos que se están llevando adelante en la región

\section{Preguntarnos:}

- ¿Qué experiencias de educación en derechos humanosinteresantes se están llevando adelante en la región que merezcan ser sistematizadas y evaluadas?

Pueden ser de educación formal o no formal, y dirigirse a distintos destinatarios en distintos niveles del sistema educativo, pero en mi criterio tienen que cumplir algunos requisitos:

○ sólida fundamentación y diseño,

- regularidad,

- duración considerable, y

- percepción de ser exitosas por parte de sus actores y destinatarios.

- ¿Cómo construir un diseño de evaluación riguroso que permita medir resultadosde estas experiencias y, sobre todo, suimpacto(efectos en el mediano y largo plazo)?

- ¿No es necesario montar el diseño de evaluación en elmismo momento en que se diseña la experiencia, de modo de garantizar las condiciones de validez desde que se inicia? En tal caso, ¿cómo nos aseguramos que las experiencias nuevas, próximas a comenzar, cuenten desde su comienzo con un plan y un diseño de evaluación?

- Aparte de los resultados y del impacto, ¿qué otros aspectos del proceso de ejecución de estas experiencias merecerían ser evaluados?

- ¿Pueden identificarse las variables que incidieron en la calidad de la experiencia y en sus buenos resultados e impacto? ¿Puedan replicarse? En otras palabras, ¿podemos extraer "lecciones aprendidas" que sean útiles a otros educadores en EDN?

\subsection{Pedagogía de la educación en dere- chos humanos}

Estimación del estado actual:

Se encuentran muchos trabajos de propuestas pedagógicas (teóricas) y, en bastante menor medida, de propuestas metodológicas. Se propone con fundamento "esto es lo que hay que hacer"; pero no se propone concretamente cómo pude hacerlo un maestro primario, un profesor de secundaria o un capacitador de servidores públicos.

Hay algunos buenos materiales y recursos latinoamericanos sobre educación en derechos humanos, por lo general concentrados en los niveles primario y secundario. Pero en la práctica no llegan con facilidad a los educadores formales (o bien porque en un cierto país no son muchos, o bien porque los que hay no tienen difusión masiva). En general, a los educadores les llegan los libros de textos oficiales o recomendados por los Ministerios de Educación. Y hay pocos libros de texto donde los temas de derechos humanos sean tratados por especialistas en derechos humanos o en educación en derechos humanos.

Ahora que cada vez más contenidos de derechos humanos se están incorporando a los currículos de la educación formal, se siente la ausencia de materiales que integren la teoría y la metodología de la educación en derechos humanos para orientar la enseñanza-aprendizaje de temas específicos de los programas de estudio dentro de un enfoque derechos humanos. Ejemplos ausentes de tal análisis y carentes de recursos apropiados son:

○ pobreza y exclusión social;

- migraciones y derechos de los migrantes;

- derechos sexuales y reproductivos;

O derechos de poblaciones LGBT;

o tratamiento de contenidos de derechos humanos por parte de otras disciplinas aparte de las ciencias sociales y la educación cívica;

- EDH para privados de libertad;

- EDH para prevenir violencia escolar y, en particular, para comunidades educativas en contextos de violencia organizada;

- EDH para niños soldados reinsertados.

Para el nivel postsecundario - universidades e institutos terciarios especializados- hay escasísimas propuestas metodológicas y materiales apropiados de enseñanza-aprendizaje.

Prioridad:

(1) Desarrollo teórico-pedagógico de temas curriculares

Profundizar el enfoque de derechos humanos sobre los diversos temas que se incluyen en el currículo de la educación formal, especialmente los menos analizados desde esta 
perspectiva (a distintos niveles educativos y para distintos destinatarios)

(2) Desarrollo de recursos didácticos

Profundizar el tratamiento didáctico de los temas curriculares: elaborar metodologías participativas y materiales de apoyo acordes.

Preguntarnos:

- ¿Cuáles son los problemas actuales en América Latina que no han sido (o han sido insuficientemente) analizados desde la perspectiva de los derechos humanos?

- ¿Existen materiales y recursos educativos para docentes y estudiantes en América Latina con un abordaje desde los derechos humanos? ¿Cuáles? ¿Podemos ubicarlos?

- En cuanto a los materiales y recursos identificados, ¿podemos recopilarlos y hacerlos accesibles a los educadores y a los formadores de educadores? ¿Cómo? (¿en base de datos en sitio web? ¿en boletines electrónicos periódicos? ¿a través de qué institución?)

- ¿Cómo podemos estimular una mayor producción teórica y didáctica en este sentido (por ej. temáticas no abordadas y destinatarios no considerados)?

\subsection{Inserción curricular}

Estimación del estado actual:

Se comprueba una creciente incorporación de contenidos de derechos humanos en asignaturas específicas del currículo de la educación formal (por lo general Educación Cívica y Ciencias Sociales). Pero no hay experiencias (o si las hay, no son conocidas) de inclusión de los derechos humanos a través de distintas disciplinas del currículo y/o espacios escolares, a pesar de que los textos teóricos de educación en derechos humanos insisten en la importancia del tratamiento transversal.

Prioridad:

Implementar la educación en derechos humanos transversalmente, cruzando distintas disciplinas $y /$ o espacios escolares (curriculares y extracurriculares).

\section{Preguntarnos:}

- ¿Cómo podemos poner en práctica la transversalidad? ¿Qué posibilidades plantea la teoría? Investigarlas (por ej. identificar temáticas pertinentes en diferentes asignaturas; organizar talleres o diseñar proyectos interdisciplinarios (de investigación o de acción comunitaria); utilizar conflictos o situaciones cotidianas como punto de partida para ensenar derechos humanos)
- ¿Cuáles de estas posibilidades resulta más apropiada o factible de poner en marcha en nuestro contexto de trabajo educativo?

- ¿Cómo nos animamos a hacer propuestas y ensayarlas? ¿Con quiénes podemos elaborar esas propuestas?

- ¿Cómo las proponemos al resto de nuestra comunidad educativa para entusiasmarlos y obtener su apoyo? (director de escuela, colegas, estudiantes, padres)

- ¿Cómo podemos evaluarlas? Proponer una metodología de evaluación desde el mismo diseño de la experiencia.

\subsection{Gestión y práctica escolar: los dere- chos humanos en la educación}

Estimación del estado actual:

La teoría y las propuestas pedagógicas sobre la educación en derechos humanos hacen referencia con frecuencia a la dimensión de "los derechos humanos en la educación" (en otras palabras, del "currículo oculto”). Nos referimos a aquellas prácticas cotidianas que no forman parte del currículo explícito pero que tienen tanto o mayor valor formativo que el primero. Sin embargo, no se les ha dado la misma reflexión y estudio que al currículo explícito. Es muy difícil decir si ha habido progresos en esta materia o no porque no contamos con estudios modélicos al respecto, ni con las herramientas de análisis para elaborarlos.(Si existen, se han realizado fuera del campo de la educación en derechos humanos y no son conocidas por los educadores en derechos humanos)

Prioridad:

Hacer diagnósticos sobre el respeto efectivo a los derechos humanos que se evidencia en la cotidianeidad de la gestión y la práctica escolar; sensibilizar a todos los actores de la comunidad educativa sobre la importancia de incorporar esta dimensión y monitorear su cumplimiento efectivo.

Preguntarnos:

- ¿Cómo podemos diagnosticar o monitorear como es el "clima o cultura de derechos humanos" en la gestión escolar y sus prácticas cotidianas, fuera de los procesos de enseñanza-aprendizaje?

- ¿Podemos construir y consensuar algunos indicadores básicos para comenzar a trabajar en esta línea?

- ¿Qué estrategias podemos utilizar para sensibilizar a los miembros de la comunidad educativa sobre esta dimensión de la educación en derechos humanos?

- Si los estudios diagnósticos y el monitoreo muestran conductas y prácticas que deben ser 
corregidas, ¿cómo gestionamos esas reformas? ¿Cómo incidimos para que se produzcan?

\subsection{Políticas públicas educativas}

Estimación del estado actual:

Pese a los considerables progresos de la educación en derechos humanos verificados en las dos últimas décadas en América Latina, seguimos teniendo una enorme asignatura pendiente: la formación de educadores. Los Ministerios de Educación, los organismos internacionales y las ONG nacionales han realizado muchos esfuerzos de capacitación de maestros en ejercicio, pero inevitablemente son breves, aislados y dispersos y alcanzan a un porcentaje ínfimo de los educadores reales. Si realmente queremos incorporar la educación en derechos humanos a la educación formal urge una acción generalizada y sistemática en este sentido, que sólo puede alcanzarse a través de políticas públicas de alcance nacional.

Prioridad:

Hacer campaña y movilizarnos de todas las maneras posibles para que la educación en derechos humanos se incorpore en la formación de los educadores.... ide una buena vez!

Construir una estrategia doble, dirigida a:

(1) Las instituciones encargadas de la formación inicial de los educadores en cada país (universidades e institutos pedagógicos)

(2) Los Ministerios o Secretarías Públicas de Educación, encargados del desarrollo profesional de los educadores en servicio.

Preguntarnos:

En cuanto a (1):

- ¿Qué podemos hacer y cómo para contribuir a incorporar la educación en derechos hu- manos en la formación inicial de educadores considerando la autonomía universitaria?

- ¿Qué estrategias conviene seguir para "auto convencer" a las autoridades universitarias de que este es un objetivo formativo crucial, especialmente a las autoridades de las escuelas de educación (a menudo conservadoras)?

- ¿Cómo incidir desde el Estado para que las universidades asuman voluntariamente esta responsabilidad que les cabe? En un caso digno de destacarse - el del Estado de Pernambuco en Brasil- se incluyó la formación en derechos humanos como un requisito en el perfil de contratación de docentes. También podría incluirse en los exámenes para contratación de docentes, en aquellos lugares donde se hagan.

- ¿Existe/n otros mecanismos que pudiera emplease?

En cuanto a (2):

- ¿Hay diagnósticos de lo que se está haciendo en este sentido en cada país? ¿Es suficiente y apropiado?

- Si no lo fuera, ¿cómo se podría mejorar?

- ¿Qué estrategias pueden seguir los especialistas individuales, los centros de investigación y formación en derechos humanos, las ONG y los organismos internacionales para que los Ministerios de Educación mejoren su oferta de desarrollo profesional en educación en derechos humanos para los educadores en servicio?

Hasta aquí llego con mis propuestas estratégicas. Quedan a la consideración y análisis de practicantes y estudiosos de la educación en derechos humanos, quienes afortunadamente son cada día más numerosos en nuestros países.

\section{Referencias}

INSTITUTO Interamericano de Derechos Humanos. III Informe Interamericano de la educación en derechos humanos: desarrollo en la formación docente. San José, Costa Rica, 2004 .

IV Informe Interamericano de la educación en derechos humanos: desarrollo en la planificación nacional. San José, Costa Rica, 2005.

$V$ Informe Interamericano de la educación en derechos humanos: desarrollo en los contenidos y espacios curriculares: 10 a 14 años.San José, Costa Rica,2006.

VI Informe Interamericano de la educación en derechos

humanos: desarrollo normativo de la educación en derechos humanos y gobierno estudiantil. San José, Costa Rica, 2007.

VII Informe Interamericano de la educación en derechos humanos: desarrollo de conocimientos específicos de derechos humanos en los programas de estudio: 10 a 14 años. San José, Costa Rica, 2008.

VIII Informe Interamericano de la educación en derechos humanos: desarrollo de conocimientos específicos de derechos humanos en los libros de texto: 10 a 14 años. San José, Costa Rica, 2009.

. IX Informe Interamericano de la educación en derechos humanos: desarrollo de la metodología de educación en derechos humanos en los libros de texto: 10 a 14 años. San José, Costa Rica, 2010.

X Informe Interamericano de la educación en derechos humanos: desarrollo en las políticas de convivencia y 
seguridad escolar con enfoque de derechos. San José, Costa Rica, 2011.

. La Consulta Interamericana sobre el estado de la Educación en Derechos Humanos en la región (20122113). In: El Derecho a la Educación en Derechos Humanos en las Américas. San José, Costa Rica, 2013.

RODINO, Ana María. La institucionalización de la educación en derechos humanos en los sistemas educativos de América Latina entre 1990 y 2012: avances, limitaciones y desafíos. In: TOSI, Giuseppe; ZENAIDE, Maria de Nazaré T.; RODINO, Ana Maria y FERNÁNDEZ,
Mónica Beatriz (Orgs.). Cultura y educación en derechos humanos en América Latina, v. I, 2014.

SEN, Amartya. Well being, Agency and Freedom: the Dewey Lectures 1984. The Journal of Philosophy, v. 82, 1985.

TOSI, Giuseppe; ZENAIDE, Maria de Nazaré T.; RODINO, Ana Maria y FERNÁNDEZ, Mónica Beatriz (Orgs.). Cultura y educación en derechos humanos en América Latina, v. I y II, Ministerio de Educación y Universidad Federal de Paraíba, Brasil, 2014.

\title{
The institutionalization of human rights education in Latin America: developments, challenges and a proposal for prioritization
}

\begin{abstract}
Taking into account the results of recent research on the incorporation of Human Rights Education (HRE) in Latin American school systems, this paper synthesizes the progresses reached during the 2000-2012period, as well as thepending gaps and shortcomings. Based on this, and in order confront the multiple challenges that lie ahead, the author recommends that experts, practitioners and institutions working in Human Rights Education in the region reach programmatic agreements, and suggests a group of strategic proposals she considers a priority due to their urgency, comprehensiveness and multiplying impact. The proposals about Human Rights Education concentrate on the areas of research, pedagogy, curriculum, school administration and practices, and public policies. The author provides a brief assessment of each area in its current state, recommends priorities for action, and poses questions of importance.
\end{abstract}

Key words: human rights education, Latin America, educational research, human rights education pedagogy, curriculum, school administration, educational public policies.

\section{A institucionalização da educação em direitos humanos na América Latina: avanços, desafios e uma proposta de prioridades}

\section{Resumo}

Tomando em consideração conclusões de investigações recentes sobre a incorporação da educação em direitos humanos nos sistemas escolares da América Latina, o artigo resume os avanços alcançados durante o período de 2000/2002, assim como as carências e limitações que subsistem. Sobre essa base e a fim de enfrentar os múltiplos desafios, a autora recomenda alcançar acordos programáticos entre especialistas, educadores e instituições que trabalham com a educação em direitos humanos na região e faz uma série de propostas estratégicas que considera prioritárias por sua urgência, integridade e impacto multiplicador. As propostas em matéria de educação em direitos humanos centram-se nas áreas de investigação, didática, currículo, gestão e prática educacional e políticas públicas. Em relação a cada área, faz-se uma breve avaliação de seu estado atual, recomendam-se prioridades de ação e se colocam perguntas as quais é importante ter respostas.

Palavras-chave: educação em direitos humanos, América Latina, pesquisa educacional, pedagogia da Educação em Direitos Humanos, currículo,gestão escolar, políticas públicas educativas. 